\title{
Nonequilibrium Translational-Rotational Effects in Nucleation
}

\author{
D. Reguera, J.M. Rubí \\ Departament de Física Fonamental, Facultat de Física, \\ Universitat de Barcelona, Diagonal 647, 08028 Barcelona, Spain
}

\begin{abstract}
The role that translational-rotational degrees of freedom play in nucleation theories is reconsidered by the introduction of a new formalism that properly accounts for the effects of motion of clusters in nucleation rate. The analysis of the non-equilibrium kinetics of the process, performed by retaining the dynamics of the clusters, enables one to clarify some of the paradoxical aspects that the inclusion of these degrees of freedom has presented.
\end{abstract}

\section{INTRODUCTION}

In spite of years of investigations and of the fact of being a ubiquitous phenomenon, nucleation is still a puzzling process not completely understood [1.2]. During the last decade, the implementation of new experiments measuring actual nucleation rates in liquid-gas systems has revealed the shortcomings of Classical Nucleation Theory (CNT) [1]. This fact has impelled the sprouting of new theories and modifications to CNT attempting to reproduce the experimental data. The corrections and improvements proposed in the literature are diverse and not always accepted with unanimity. Examples of these corrections are based on [3]: self-consistency considerations; contribution of translational, rotational and vibrational degrees of freedom; curvature effects in the surface tension; monomer depletion and cluster scavenging [4] or even the inclusion of effects associated to spatial [5] or temperature inhomogeneities [6].

A very controversial issue in nucleation theory is the proper accounting for embryo degrees of freedom, sometimes referred to as the translational-rotational paradox. The controversy dates back to the consideration of Lothe and Pound [7] of what appears to be a serious inconsistency in the conventional theory of nucleation from the vapor phase. These authors pointed out that several important contributions to the free energy of formation of the critical cluster attributable to rotational and translational degrees of freedom had been neglected in CNT. The inclusion of these additional terms in the way they proposed increased the nucleation rate by a factor on the order of $10^{17}$, enough to destroy reasonable agreement between theory and experiments.

Reiss and co-workers 8.9.9 attempted to resolve the paradox by arguing that experimental surface tension in the capillarity approximation already takes into account the rotational and most of translational effects. From this point of view, Reiss-Katz-Cohen theory presents a new way to incorporate these effects into CNT, leading to a much smaller change in the expected rates, between $10^{3}$ and $10^{6}$.

There followed a series of counter arguments and discussions by several authors 10. Although there has been a long standing controversy, the issue was never satisfactorily resolved and the proper inclusion of these contributions continued being debated [11. The issue seems to have come to an end with the recent work of Reiss, Kegel and Katz [12] which apparently constitutes the proper resolution of the paradox and clarifies other inconsistencies of the nucleation theory.

As we have seen, considerable emphasis has been put on the introduction of the translational and rotational degrees of freedom in the equilibrium partition function of a nucleating droplet. However, these corrections refer only to the influence of these degrees of freedom in the equilibrium sense. Translational and rotational degrees of freedom also arise from the motion and rotation of the clusters through the metastable phase. This is a purely non-equilibrium effect, which may have a relevant influence in the nucleation kinetics.

Our purpose in this paper is precisely to present a new theory to consistently incorporate translational and rotational contributions due to cluster motion in nucleation. Contrarily to the theories proposed up to now, we will adopt a non-equilibrium description more proper for a problem intrinsically out of equilibrium as nucleation is. In this sense, we will show that when considering nucleation retaining the dynamics of the problem a new and important correction to the nucleation kinetics arises.

The paper is distributed as follows. In Sec. II we analyze the influence of the translational/rotational degrees of freedom in the nucleation process and review how these degrees of freedom have been considered from the equilibrium point of view. To analyze the potential non-equilibrium effects of the motion of the clusters in nucleation, we derive in Sec. III, the equation governing the kinetics of the nucleation, retaining the dynamics of the clusters. After a proper elimination of the velocity variables, this equation will be used in Sec. IV to obtain a nonequilibrium correction to the nucleation rate, discussing explicitly the results for nucleation rates of water. Finally, in the Conclusions, we analyze and summarize the main consequences of our work. 


\section{INFLUENCE OF TRANSLATIONAL-ROTATIONAL DEGREES OF FREEDOM IN NUCLEATION}

Phenomenological theories of nucleation are based on the formulation of the free energy $G(n)$ associated to the formation of a cluster of $n$ molecules from the metastable phase. The expression proposed by CNT, based on capillarity approximation, is obtained by treating the cluster as a macroscopic spherical droplet with bulk and surface free energy contributions

$$
G(n)=-k_{B} T n \ln S+\sigma s_{1} n^{2 / 3}
$$

Here $T$ is the temperature, $S$ the saturation ratio, $\sigma$ the surface tension, $s_{1}$ the mean surface of a single molecule of the liquid phase and $k_{B}$ is the Boltzmann constant. However, treating embryos as macroscopic objects and using thermodynamic arguments to calculate the work of embryo formation give rise to important inconsistencies [1, 3 . Capillarity approximation leave out translational, rotational and configurational contributions; it predicts a non-zero value for the energy of monomer formation $(G(1) \neq 0)$ and ignores possible dependencies of surface tension on the temperature or the radius of the clusters. In spite of all of these inconsistencies, the results predicted by $\mathrm{CNT}$ are in most cases in reasonable agreement with experimental data. Paradoxically, it seems that most of the corrections originally introduced to restore the logical consistency of CNT spoil this agreement and leads to worst results.

Basically, the corrections proposed by these theories in liquid-vapor systems, can be recovered from the general formula [13]

$$
\frac{G(n)}{k_{B} T}=-n \ln S+k_{n} \frac{\sigma s_{1}}{k_{B} T} n^{\gamma}+\tau \ln n+\ln \left(V q_{0}\right)
$$

where $V$ is the volume, and $k_{n}, \gamma, \tau$ and $q_{0}$ are parameters whose expressions and values are different for each theory. In this expression, the first and second terms are the bulk and surface contributions, respectively, the two contributions considered in CNT. The remaining terms include rotational, translational, vibrational, configurational, and replacement contributions related to additional degrees of freedom which capillarity approximation may leave out. Our analysis will be focused on translational-rotational aspects of nucleation.

Capillarity approximation deals with the free energy required to form a single cluster at rest in the metastable phase. However, the conception of droplets at rest constitutes an approximation to the real behavior of the system. The small clusters which are the embryos of the new phase can spontaneously appear at any point of the system, and with arbitrary orientation. Moreover, due to the mesoscopic size of these entities, they move and spin around the metastable phase because the influence of the medium in which they are embedded (Brownian motion). Both factors constitute translational and rotational degrees of freedom of the cluster and must be taken into account to describe accurately the nucleation process. But the influence and the way of considering both effects is different. The arbitrariness of positions and orientations which a cluster may occupy can be incorporated as a purely equilibrium correction by including these translational and rotational degrees of freedom in the partition function of the cluster. Consequently, this yields a modification of the free energy of formation of this cluster. Contrarily, the effect of the movement and rotation of the clusters does not alter the nucleation barrier itself. It is a nonequilibrium factor that modifies the kinetics of the process.

Most of the work on translational-rotational correction, and the paradox itself, addresses to modify the free energy to form a cluster of size $n$, taking into account these degrees of freedom in the partition function. In this sense, one may refer to the works of Lothe and Pound (LP) [7], and the more recent and accurate theory of Reiss, Kegel and Katz (RKK) 12]. These works deal with purely equilibrium corrections. The common underlying idea is that if nucleation occurs in a volume $\mathrm{V}$, the physical critical cluster may appear anywhere in the system with any orientation. Considering that all these positions of the cluster are equivalent, one then has this additional translational and rotational freedom to account for in the partition function. The accounting of this degrees of freedom increases the value of the partition function, consequently reducing the free energy of formation of a droplet and thus increasing the nucleation rate.

The difference between both treatments and the origin of the paradox concerns what is the proper accounting of this degrees of freedom in the partition function and how to define and distinguish different states of a cluster in a volume V. Using the CNT expression for the free energy of formation of a cluster entails the extrapolation of a macroscopic expression to the mesoscopic scale. Whereas it is clear how to locate one macroscopic droplet at rest, the characterization becomes less obvious when this cluster only contains a small number of molecules. For instance, the position of a mesoscopic cluster constituted by $n$ molecules inside a spherical volume $v$, can be described in terms of the position of its center of mass, or by the position of the center of the spherical container. Whereas for a macroscopic droplet both description practically coincide, for the mesoscopic one they differ due to the fluctuations.

The other problem arising in the mesoscopic scale concerns the pertinent volume scale one has to use to discretize the space with the purpose of distinguishing different states in the calculation of the partition function. The uncertainty 
in defining a volume scale is a well-know problem in Statistical Mechanics [14]. For macroscopic systems, however, this arbitrariness of the length scale is not relevant, because only introduces a logarithmic correction to the free energy which is always negligible in the thermodynamic limit. But for a small embryo, the correction may become important. Hence the problem and the central point in the paradox is to know what is the proper and physical meaningful volume scale to count states in the partition function and consequently to calculate the free energy of a mesoscopic cluster.

A reasonable choice for the volume scale would be of course $\Lambda^{3}$, based on the de Broglie wavelength scale $\Lambda$; that was the one used by Lothe and Pound [7]. They assumed that the location of a spherical cluster of $n$ molecules is defined through the position of its center of mass and its orientation. Therefore every configuration of $n$ molecules inside a spherical volume $v$ with different position of the center of mass and different orientation constitute a different realization of the same cluster and consequently a new state to be accounted for in the partition function. The minimum uncertainty to locate and distinguish different states is thus the quantum uncertainty. That is, every configuration of $n$ molecules whose center of mass and orientation differs by $\Lambda$ and $\Lambda_{\text {rot }}$, respectively is a new state that increases the value of the partition function. This criterion leads to the following correction of the free energy

$$
\Delta G_{L P}(n)=-k_{B} T \ln \left(\frac{V}{\Lambda^{3}} \frac{8 \pi^{2}}{\Lambda_{r o t}^{3}} \frac{1}{q_{r e p}}\right)
$$

where $\Lambda=\left(\frac{h^{2}}{2 \pi m_{1} k_{B} T n}\right)^{1 / 2}$, and $\Lambda_{\text {rot }}=\left(\frac{h^{2}}{2 \pi I k_{B} T}\right)^{1 / 2}$, with $m_{1}$ the mass of a single monomer, $I$ the moment of inertia of the cluster, $h$ the Planck's constant, and $q_{\text {rep }}$ a "replacement factor" related to the entropy reduction accompanying the separation of $n$ molecules from the system. Assuming reasonable values for vapor condensation, the small size of the de Broglie lengths, originates that these additional terms increase nucleation rates by approximately $10^{17}$, destroying in most cases the agreement between CNT results and experimental data.

But the de Broglie length is not the only reasonable scale one may choose, and RKK theory [12] proposes a different alternative. From their point of view, the location of a cluster must be defined by the position of the spherical container, and not by its center of mass. All the possible configurations of $n$ molecules inside a spherical container of volume $v$ correspond then to the same cluster and consequently are already accounted for in the CNT expression of the free energy. In particular, all the possible orientations of the molecules inside the spherical volume are included, which implies that the rotational degrees of freedom are already accounted for in the CNT free energy barrier. In addition, to incorporate the translational degrees of freedom one has to take into account that not all the different positions which this spherical cluster may occupy inside the volume $\mathrm{V}$ correspond to different clusters. If we displace the spherical container a small distance $d x$, most of the configurations of the $n$ molecules compatible with the new situation are the same and have been already accounted for in the undisplaced original cluster. Hence treating this new position of the cluster as a different cluster entails an overcounting of states in the partition function. They affirm that this is essentially the origin of the excessively high correction of obtained by LP, the overcounting of configurations in choosing the quantum length scale to count states in the partition function. Consequently, there exist a minimum volume scale to differentiate clusters in the configurational space. For a nearly incompressible drop, this scale turns out to be the one related to the volume fluctuation $\sqrt{k_{B} T \kappa v_{1} n}$. That is, a cluster cannot be located more precisely than its volume fluctuation. This criteria prevents the overcounting of configuration and gives rise to a correction in the nucleation barrier

$$
\Delta G_{R K K}=-k_{B} T \ln \frac{V}{\sqrt{k_{B} T \kappa v_{1} n}}
$$

leading to a much smaller change in nucleation rates of the order of $10^{4}$.

Both LP and RKK approaches are deeply rooted in equilibrium statistical arguments. However, the correction they propose does not in fact concern the movement of clusters. Different ways exist by which this movement can influence the process.

On one hand, the movement of the clusters through the metastable phase may alter the rate at which the cluster incorporates molecules of the metastable phase. Olson and Hamill [15] considered the effect of the movement of clusters in the supersaturated vapor in the rate at which a cluster gains monomers $\beta(n)$. Yet, the correction to $\beta(n)$ has been shown to be negligible [3].

But on the other hand, the movement of the cluster can modify not only the rate of growth of a cluster, but also the kinetics of the whole process. Contrarily to the equilibrium point of view, our objective will be to analyze the influence of the movement and rotation of clusters in the nucleation process from a dynamical point of view. Nucleation is a nonequilibrium kinetic phenomenon essentially related to the variation of the size distribution of clusters present in the system. If the clusters are moving through the metastable phase, its movement may influence the evolution of the cluster size distribution thus altering the kinetics of the process. Therefore, it seems reasonable to study the process not only by considering the evolution of the size of clusters but also retaining its dynamics. This is the approach we develop in the next section. 


\section{NONEQUILIBRIUM KINETICS OF NUCLEATION}

We will model the real system, composed by droplets and molecules of the metastable phase, as a dispersion of clusters of different and varying sizes. Since we will focus on homogeneous nucleation in spatial homogeneous systems, the spatial variables are not relevant. Thus, the variables characterizing a cluster are its size $n$, its velocity $\vec{u}$ and its angular velocity $\vec{\omega}$. The description of the system will be carried out in terms of the distribution function $f(\underline{\Gamma}, t)$, with $\underline{\Gamma} \equiv(n, \vec{u}, \vec{\omega})$, whose evolution is dictated by the continuity equation

$$
\frac{\partial f(\underline{\Gamma}, t)}{\partial t}=-\frac{\partial}{\partial \underline{\Gamma}} \cdot \underline{J}(\underline{\Gamma}, t)
$$

where $\underline{J} \equiv\left(J_{n}, \vec{J}_{u}, \vec{J}_{\omega}\right)$ is the current of cluster sizes and velocities defined in $\underline{\Gamma}$-space.

The nucleation process may be conceived as a diffusion process in the space spanned by the values of $\underline{\Gamma}$ through the energy barrier

$$
C(\underline{\Gamma})=\Phi(n)+\frac{1}{2} m(n) u^{2}+\frac{1}{2} I(n) \omega^{2},
$$

where $\Phi(n)$ represents the energy of formation of a $n$-cluster at rest of mass $m(n)=n m_{1}$ and moment of inertia $I(n)$. For a spherical rigid cluster $I(n)=\frac{2}{5} m(n) r^{2}$, thus implying $I(n) \sim n^{5 / 3}$. The remaining terms correspond to translational and rotational kinetic energies.

The entropy of the system, $S(t)$, is given through the Gibbs entropy postulate 16,17

$$
S(t)=-k_{B} \int f(\underline{\Gamma}, t) \ln \frac{f(\underline{\Gamma}, t)}{f_{e q}(\underline{\Gamma})} d \underline{\Gamma}+S_{e q}
$$

where $S_{e q}$ is the value of the entropy at equilibrium. Its variations can be expressed in the form

$$
\delta S(t)=-\frac{1}{T} \int \mu(\underline{\Gamma}, t) \delta f(\underline{\Gamma}, t) d \underline{\Gamma}
$$

where $\mu(\underline{\Gamma}, t)$ is a generalized chemical potential defined in $\underline{\Gamma}$-space.

$$
\mu(\underline{\Gamma}, t)=k_{B} T \ln \frac{f(\underline{\Gamma}, t)}{f_{e q}(\underline{\Gamma})}+\mu_{e q}
$$

This expression corresponds to the chemical potential of an ideal, i.e. non-interacting, dispersion of clusters in the liquid phase. In the latter equation, $f_{e q}(\underline{\Gamma})$ represents the equilibrium distribution given by

$$
f_{e q}(\underline{\Gamma}) \propto \exp \left(-\frac{C(\underline{\Gamma})}{k_{B} T}\right)
$$

and $\mu_{e q}$ is the chemical potential at equilibrium.

The expression for the current $\underline{J}$ defined in Eq. 6 can be obtained from nonequilibrium thermodynamics. The entropy production

$$
\sigma=-k_{B} \int \underline{J} \cdot \frac{\partial}{\partial \underline{\Gamma}} \ln \frac{f}{f_{e q}} d \underline{\Gamma}
$$

follows from Eqs. (4), (7) and (8). The resulting linear law, obtained from the assumption of isotropy and locality in [-space [18], is given by

$$
\underline{J}(\underline{\Gamma}, t)=-\underline{L} \cdot \frac{\partial}{\partial \underline{\Gamma}} \ln \frac{f}{f_{e q}}
$$

where $\underline{L}$ is the corresponding matrix of Onsager coefficients. This expression can be used in the continuity equation (画) thus leading to the Fokker-Planck equation

$$
\frac{\partial f}{\partial t}=\frac{\partial}{\partial \underline{\Gamma}} \cdot\left(\underline{D} \cdot \frac{\partial f}{\partial \underline{\Gamma}}+\frac{\underline{D}}{k_{B} T} \cdot \frac{\partial C}{\partial \underline{\Gamma}} f\right)
$$


where $\underline{D}=\frac{\underline{L}}{T f}$ is the diagonal matrix of diffusion coefficients.

The former equation provides a complete dynamical description of the system in terms of the variables $n, \vec{u}$ and $\vec{\omega}$. However, from the analysis of the time scales of the system it is easy to realize that in the nucleation time scale the system reaches equilibration in velocities space. The characteristic nucleation relaxation time [6] is given by $\tau_{N} \approx \frac{1}{\beta(n)}$ where for the rate $\beta(n)$ of addition one molecule to a droplet of size $i$ we may adopt the classical expression taken from kinetic theory of gases $\beta(n)=\frac{p}{\sqrt{2 \pi m_{1} k T}} s_{1} n^{2 / 3}$.

Similarly, the Brownian translational and rotational time scales are $\tau_{\text {trans }} \approx \frac{m}{6 \pi \eta a}$ and $\tau_{\text {rot }} \approx \frac{I}{8 \pi \eta a^{3}}$ respectively , where $m$ is the mass of the Brownian particle, $a$ is its radius and $\eta$ is the viscosity. For nucleation in liquid-vapor systems, the order of magnitude of these characteristic times is approximately $\tau_{N} \approx 10^{-8} n^{2 / 3}$ and $\tau_{\text {trans }}, \tau_{\text {rot }} \approx$ $10^{-13} n^{2 / 3}$. Therefore a clear separation between time scales exists, the condition $\tau_{N} \gg \tau_{\text {trans }}, \tau_{\text {rot }}$ holds, and one can perform an adiabatic elimination of the fast variables (the velocities) [19], assuming that they reach their equilibrium distribution.

After performing the adiabatic elimination of velocities, the resulting dynamics is governed by the Fokker-Planck equation

$$
\begin{aligned}
\frac{\partial f(n, t)}{\partial t}=\frac{\partial}{\partial n}( & \bar{D}_{N}(n) \frac{\partial f}{\partial n}+ \\
& \left.+\frac{\bar{D}_{N}(n)}{k_{B} T}\left(\frac{d \Phi(n)}{d n}+4 k_{B} T \frac{1}{n}\right) f\right)
\end{aligned}
$$

We may now identify the diffusion coefficient $\bar{D}_{N}(n)$ with the forward rate $\beta(n)$, and obtain the expression for the effective nucleation barrier $\Delta G(n)$

$$
\Delta G(n)=\Phi(n)+4 k_{B} T \ln n
$$

The conclusion is that translational and rotational motion of clusters in the spatial homogeneous medium introduces an effective additional size-dependent contribution $4 k_{B} T \ln n$ in the nucleation barrier, inherent to the diffusion process and thus independent of the energy of formation of cluster at rest $\Phi(n)$. It is important to highlight that since this contribution is always positive translational-rotational motion always increases nucleation barrier and consequently reduces nucleation rates, contrarily to the results of the equilibrium corrections which always decrease the nucleation barrier thus increasing the rate. One may wonder about the reasons for this feature. In essence, the difference lies on the treatment of velocity variables.

From the equilibrium point of view, it was always assumed that the velocity and the motion of the clusters does not influence the nucleation process. And the justification was precisely the same that the one we have used to eliminate the velocity variables in our Fokker-Planck equation. The time scale of the velocity relaxation is much faster than the nucleation time scale, so it was assumed that nucleation occurs when velocity distribution is equilibrated thus not influencing the process. Following this line of reasoning, the free energy of formation of clusters is constructed by averaging the contribution of all cluster velocities. The nucleation kinetics is then studied focusing only on the evolution of the cluster size distribution.

In contrast with these lines of reasoning, in our treatment we consider that, due to the coupling between the dynamics of $n$ and $(\vec{u}, \vec{\omega})$, the elimination of velocities must be performed at the last stage because it influences the kinetics of the whole process. Thus we maintain velocities of a cluster as relevant variables in the description of the process. Instead of constructing an averaged free energy of a drop taking into account all possible velocities, we directly use the free energy to form a $n$-cluster with velocities $\vec{u}, \vec{\omega}$ (Eq. 5) to study the evolution of the system as well with respect to the size of clusters as their velocities. Finally, we perform an adiabatic elimination of $\vec{u}$ and $\vec{\omega}$ from characteristic time scales considerations. The result is a net positive contribution to the nucleation barrier.

In both cases, the velocity is finally eliminated using the same equilibrium distribution and the same arguments. One then may wonder what is the physical origin of the additional effective contribution we obtain. The underlying reason can be naively understood as follows. Although the velocity is initially equilibrated and equilibrates very fast, every time that a cluster gains or looses a molecule not only the size distribution of the clusters is altered, but also the velocity distribution. Therefore, the alteration of cluster size distribution entails a reorganization of velocities distribution through diffusing currents. These currents involve an additional dissipation which has an energetic cost. Consequently, the growing of the cluster requires more effective energy, because part of that is expended in the reorganization (in the new equilibration) of the velocity distribution. As we will show in the following section, the nucleation rate is thus slowed down. 


\section{NUCLEATION RATE}

The consideration of translational and rotational contribution due to the motion of the clusters in the nucleation process then leads to the following expression for the stationary nucleation rate [20], derived from Eq. (13)

$$
J=A^{*} \exp \left(-\frac{\Delta G\left(n^{*}\right)}{k_{B} T}\right)
$$

where $n^{*}$ is the size of the critical nucleus obtained from the condition of maximum of the nucleation barrier

$$
\Phi^{\prime}\left(n^{*}\right)+4 k_{B} T \frac{1}{n^{*}}=0
$$

and $A^{*}$ is the pre-exponential factor which for liquid-gas nucleation is given by

$$
A^{*}=\beta\left(n^{*}\right) \frac{1}{V} \sqrt{-\frac{1}{2 \pi k_{B} T} \frac{\partial^{2} \Delta G\left(n^{*}\right)}{\partial n^{2}}}
$$

In order to quantitatively illustrate the effect of our correction in the nucleation rate, we will particularize our general formulation to a concrete physical situation by using a specific model for the energy barrier $\Phi(n)$ of formation of clusters at rest. Our aim in this sense is to show how the consideration of the translational-rotational degrees of freedom in the way we propose may lead some phenomenological theories to a better agreement with experimental data for some substances.

As a concrete example we will focus on the nucleation rates of water, comparing with the experimental results of Viisanen et. al. [21]. As a model for the free energy of formation of a cluster at rest $\Phi(n)$, we will use RKK model, explicitly

$$
\Phi_{R K K}(n)=-k_{B} T n \ln S+\sigma s_{1} n^{2 / 3}-k_{B} T \ln \frac{V}{\sqrt{k_{B} T \kappa v_{1} n}}
$$

Data for the compressibility of supercooled water are taken from Ref. 22]. As we have discussed in Section II, the RKK model constitutes an improvement of the CNT in the sense that solves some consistency problems and properly incorporates the equilibrium corrections due to the translational degrees of freedom. Unfortunately, this theoretical consistency does not guarantee an improvement of the quantitative results. In fact, for the particular case of water, the RKK theory clearly overestimates the nucleation rate by factor of $10^{5}-10^{6}$, whereas the discrepancy of the CNT is approximately two orders of magnitude (see Fig. 1).

However, taking into account the nonequilibrium effects of the motion of the cluster in the way we propose and using the RKK energy barrier one can achieve a very good concordance with the experimental results. In Fig. 1, we compare the experimental values of Ref. [21] for the nucleation rate of water at different temperatures, with the predictions of CNT, and the values obtained with our theory from Eq. 15. We can see that the agreement of our result is excellent, specially at high temperatures. For low temperatures, the results get slightly worst probably because the low temperature anomalies of the compressibility of the supercooled water (in fact it diverges at approximately $228 \mathrm{~K}$, see Ref [22]). Nevertheless, the maximum discrepancies are less than a factor of 3 for the whole range of temperatures above $228 \mathrm{~K}$. This example illustrates the fact that an energy barrier constructed under the requirement of preserving self-consistency and including properly the nonequilibrium effects may reproduce experimental results.

It is important to emphasize that the accuracy of the results we may provide mostly relies on the correctness of the energy barrier at rest $\Phi(n)$ we adopt. The theory we present aims to analyze the nonequilibrium effects of the motion of the clusters in nucleation, but phenomenological nucleation barriers based on capillarity approximation present other serious drawbacks, like self-consistency considerations, or dependencies of the surface tension on the radius of cluster or temperature, on which agreement with experiments depends on. It is evident, that in this cases translational corrections are not sufficiently important to reestablish in those cases agreement with experiments.

On what concerns nucleation in liquids, there is a general belief that cluster motion is of little influence and does not need to be considered. Homogeneous nucleation of crystals is much less well understood than condensation discussed previously, and presents additional difficulties [23]. The first one is related with the lack of a kinetic approach to estimate the pre-exponential factor $A^{*}$ or even the forward rate $\beta(n)$. The second difficulty concerns the absence of independent measurements of the solid-liquid surface free energy in the undercooled regime. In fact, the surface free energy is mostly evaluated from nucleation rate experiments as a fitting parameter. Thus, we cannot perform direct tests of nucleation rate experiments against crystal nucleation theories. 
However, in spite of the differences between gas and crystal nucleation, and the additional problems that the latter presents, the formalism previously developed to introduce translation-rotation effects in liquid-gas nucleation remains applicable. Consequently, the net effect of translational-rotational degrees of freedom predicted by our model is again the variation of the energy barrier given in Eq. (14). In this case, the differences between our nonequilibrium treatment and the equilibrium approaches become even more obvious, because in condensed phases translational/rotational contributions to the free energy of a cluster (in the partition function) are expected to be negligible [23], while the influence of the movement of clusters in the kinetics of the process still remains the same.

\section{CONCLUSIONS}

In summary, in this paper we have reconsidered the role played by the translational-rotational degrees of freedom through the introduction of a formalism that properly accounts for the effects of motion of clusters in nucleation rate. The analysis of the problem has been performed by taking into account the fact that the emerging clusters remain embedded in the metastable phase and that their movement could induce modifications in the nucleation rate. Actually, the motion of the clusters influence the kinetics of size evolution of clusters, therefore the study of the problem must be performed retaining their dynamics. The influence of the medium is then reflected in the appearance of a new contribution to the energy barrier which is independent of the definition of cluster and of the energy barrier at rest one adopts. The contribution we have found originates from Brownian diffusion of clusters and in this sense is a translational contribution arising from a nonequilibrium situation and not from equilibrium statistical considerations. The effect we have introduced is fundamental as is due to the ineluctable presence of the medium during the nucleation process.

The conclusion is that the motion of the clusters is relevant in the kinetics of nucleation, leads to a reduction of the nucleation rates, and its proper inclusion constitutes a first step toward the construction of a nucleation energy barrier that preserve all logical consistencies and is able to reproduce and predict experimental results.

The revision we propose is unable by itself to correct all possible disagreements between theories and experimental results. The field is still open, and more investigations are needed, specially the ones aimed to find out a better expression for the energy of formation of clusters at rest. When this objective is achieved, it still will be essential to include the influence of the medium in order to obtain a prediction suitable to be contrasted with experiments. The presence of the medium should then be taken into account in future developments of nucleation theories.

A methodological aspect to be emphasized is that our analysis, leading to kinetic equations of the Fokker-Planck type, could be directly extended to consider other hydrodynamic effects in nucleation processes, as the ones related to the presence of gradients or inhomogeneities. We then provide a theoretical framework from which the influence that the proper dynamics of the metastable phase may play in the nucleation process could be studied systematically.

\section{ACKNOWLEDGMENTS}

We would like to thank T. Alarcón, A. Pérez-Madrid, H. Reiss, R. Bowles and Y. Djikaev for fruitful discussions, and R. Strey for sending us their revised experimental data. This work has been supported by DGICYT of the Spanish Government under grant PB98-1258. D. Reguera wishes to thank Generalitat de Catalunya for financial support.

[1] A. Laaksonen, V. Talanquer and D. W. Oxtoby. Annu. Rev. Phys. Chem. 46, 489 (1995).

[2] P. G. Debenedetti, Metastable Liquids. Concepts and Principles (Princeton University Press, Princeton, NJ, 1969), Chap. 3.

[3] D. T. Wu. Solid State Phy. 50, 37 (1996).

[4] G. Shi and J. H. Seinfeld. J. Chem. Phys. 92, 687 (1990).

[5] G. Shi and J. H. Seinfeld. J. Appl. Phys. 68, 4550 (1990)

[6] B. E. Wyslouzil and J. H. Seinfeld. J. Chem. Phys. 97, 2661 (1992).

[7] J. Lothe and G. M. Pound. J. Chem. Phys. 36, 2080 (1962).

[8] H. Reiss and J. L. Katz. J. Chem. Phys. 46, 2496 (1967).

[9] H. Reiss, J. L. Katz, and E. R. Cohen. J. Chem. Phys. 48, 5553 (1968). 
[10] J. Lothe and G. M. Pound. J. Chem. Phys. 48, 1849 (1968); R. Kikuchi, J. Stat. Phys. 1, 351 (1969); J. Lothe and G. M. Pound, in Nucleation (A. C. Zettlemoyer, ed.) p. 109, Marcel Dekker, New York (1969); K. Nishioka and G. M. Pound. Adv. Colloid Interface Sci. 7, 205 (1977); H. Reiss. Adv. Colloid Interface Sci. 7, 1 (1977); R. Kikuchi. Adv. Colloid Interface Sci. 7, 67 (1977).

[11] V. Ruth, J. P. Hirth and G. M. Pound. J. Chem. Phys. 88, 7079 (1988).

[12] H. Reiss, W. K. Kegel, and J. L. Katz. Phys. Rev. Lett. 78, 4506 (1997); J. Phys. Chem. A 102, 8548 (1998).

[13] C. S. Kiang, D. Stauffer, G. H. Walker, O. P. Puri, J. D. Wise, Jr. and E. M. Patterson. J. Atmos. Sci. 28, 1222 (1971).

[14] T.L. Hill. Statistical Mechanics(Dover, New York, 1987).

[15] T. Olson and P. Hamill. J. Chem. Phys. 104, 210 (1996).

[16] J. M. Rubí and P. Mazur. Physica A, 250, 253 (1998).

[17] D. Reguera, J. M. Rubí and A. Pérez-Madrid. J. Chem. Phys. 109, 5987 (1998).

[18] S. R. de Groot and P. Mazur, Non - Equilibrium Thermodynamics, (Dover, New York, 1984).

[19] H. Risken, The Fokker - Planck Equation (Springer, Berlin, 1984).

[20] J. E. McDonald, Am. J. Phys. 30, 870 (1962) and 31, 31 (1963).

[21] Y. Viisanen, R. Strey and H. Reiss. J. Chem. Phys. 99, 4680 (1993) and J. Chem. Phys. 112, 8205 (2000).

[22] R.J. Speedy and C.A. Angel. J. Chem. Phys. 65, 851 (1976).

[23] K. F. Kelton, Solid State Phy. 45, 75 (1991). 


\section{FIGURE CAPTIONS}

- Figure 1.- Comparison of experimental nucleation rates $J$ as a function of the supersaturation $S$ with the predictions of the Classical Nucleation Theory (dashed lines) and the new theory (full lines). Crosses represent data of Viisanen et. al. [21 for water at different nucleation temperatures. 


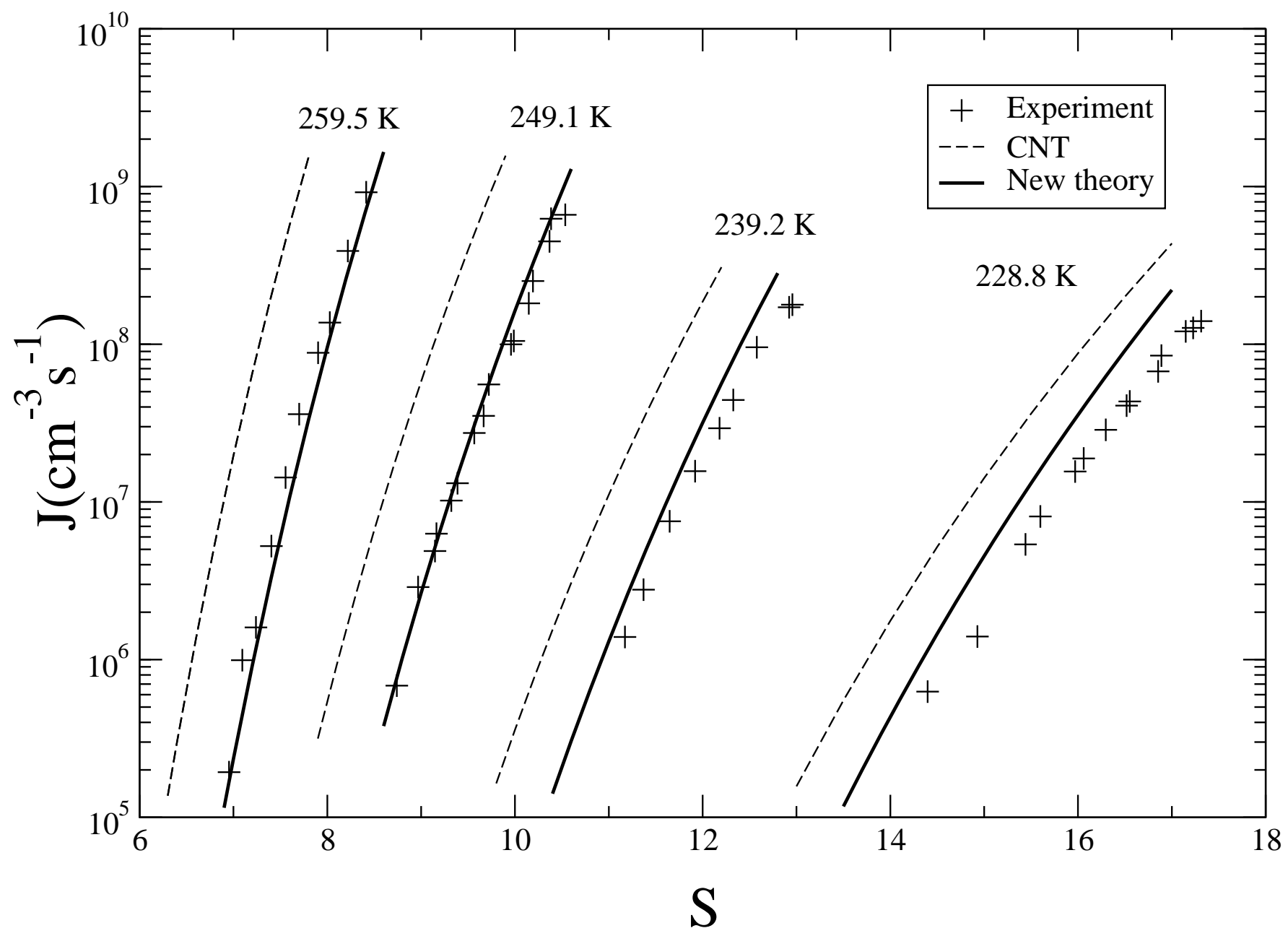

FIG. 1. D. Reguera, J.M. Rub. 